\title{
Inhalation Vapor, Ointment Dosage Form
}

National Cancer Institute

\section{Source}

National Cancer Institute. Inhalation Vapor, Ointment Dosage Form. NCI Thesaurus.

Code C149588.

Semi-solid preparation consisting of an ointment intended for generation of vapor to be inhaled to obtain a local effect. The vapor may be generated by adding the ointment to hot water. 